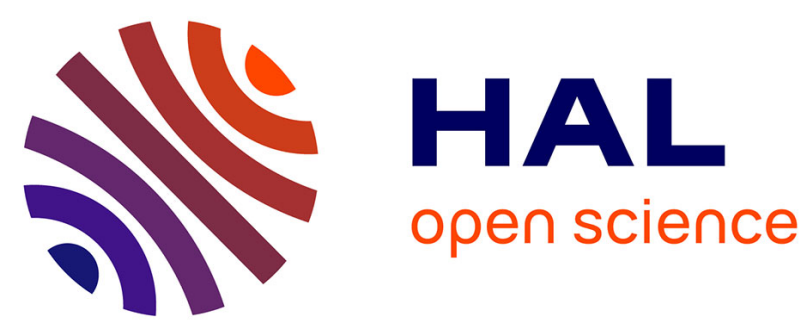

\title{
About the Numerical Robustness of Biomedical Benchmark Cases: Interlaboratory FDA's Idealized Medical Device
}

Vladeta Zmijanovic, Simon Mendez, Vincent Moureau, Franck Nicoud

\section{To cite this version:}

Vladeta Zmijanovic, Simon Mendez, Vincent Moureau, Franck Nicoud. About the Numerical Robustness of Biomedical Benchmark Cases: Interlaboratory FDA's Idealized Medical Device. International Journal for Numerical Methods in Biomedical Engineering, 2017, 33 (1), pp.e02789. 10.1002/cnm.2789 . hal-01290017

\section{HAL Id: hal-01290017 https://hal.science/hal-01290017}

Submitted on 2 Apr 2016

HAL is a multi-disciplinary open access archive for the deposit and dissemination of scientific research documents, whether they are published or not. The documents may come from teaching and research institutions in France or abroad, or from public or private research centers.
L'archive ouverte pluridisciplinaire $\mathbf{H A L}$, est destinée au dépôt et à la diffusion de documents scientifiques de niveau recherche, publiés ou non, émanant des établissements d'enseignement et de recherche français ou étrangers, des laboratoires publics ou privés. 


\title{
About the Numerical Robustness of Biomedical Benchmark Cases: Interlaboratory FDA's Idealized Medical Device*
}

\author{
Vladeta Zmijanovic ${ }^{1 *}$, Simon Mendez ${ }^{1}$, Vincent Moureau ${ }^{2}$ and Franck Nicoud ${ }^{1}$ \\ ${ }^{1}$ Institute of Montpellier Alexander Grothendieck, CNRS - UMR5149 \& University of Montpellier, \\ 34095 Montpellier, France \\ ${ }^{2}$ CORIA, CNRS, INSA 8 University of Rouen, 76801 Saint-Etienne-du-Rouvray, France
}

\begin{abstract}
The need for reliable approaches in numerical simulations stands out as a critical issue for the development and optimization of cardiovascular biomedical devices. This led the United States Food and Drug Administration to undertake a program of validation of computational fluid dynamics methods for transitional and turbulent flows. In the current investigation, large-eddy simulation is used to simulate the flow in the first benchmark medical device and results are confronted to the existing laboratory experiments. This idealized medical device has the particularity to feature transition to turbulence after a sudden expansion. The effects of numerical parameters and low-level inlet perturbations are investigated. Results indicate a considerable impact of numerical aspects on the prediction of the location of the transition to turbulence. The study also demonstrates that injecting small perturbations at the inflow greatly improves the streamwise velocity estimation in the transition region and substantially contributes to the robustness of the flow statistical data.
\end{abstract}

\section{Introduction}

Cardiovascular biomedical devices that feature complex geometries are common in medical practice. For the design and optimization of such devices, computational fluid dynamics (CFD) has been used in recent decades as a tool of choice $[1,2]$. The great potential of CFD lies in its ability to effectively predict the flow field via accurate estimation of flow quantitative data and in many cases to overcome or complement the physical limitations of experimental diagnostics, providing the full insight at an affordable cost.

However, in some cases, accuracy and reliability of commonly used CFD approaches are flawed [3]. This is especially prejudicial when quantities of interest are derived from the primary variables (velocity, pressure etc.). That is the case for shear stress, which is relevant to compute mechanical blood damage in ventricular assist devices (VAD) [2, 4], but also in blood flow through the stents, pumps, grafts, in/out of the storage containers, etc. In a number of medical devices, blood is subjected to flow pulsations, pumping, fluid-wall interactions and irregular inlets and outflows. All of these conditions affect blood flow, which may reach regimes where it undergoes turbulent transitions, increasing the difficulty of the prediction.

Occurrence of the turbulence in blood flows has a significant effect on shear, energy losses and transport. Failure of CFD solvers to predict turbulence in blood flows can strongly impact the results and may lead to critical erroneous conclusions.

\subsection{FDA medical nozzle benchmark model and current state of the art}

In the recent years, the United States Food and Drug Administration (FDA) has proposed a series of unified benchmarking test cases to assess the accuracy of CFD methods [5]. The first

*Paper published in: Int. J. Numer. Meth. Biomed. Engng. 2016; http://dx.doi.org/10.1002/cnm.2789 
benchmark case represents an idealized medical nozzle device which incorporates many critical flow features that are typically encountered in cardiovascular medical devices. The objective of this benchmark is to validate CFD approaches against several series of FDA's multi-laboratory experiments, for different flow regimes, from laminar to fully turbulent.

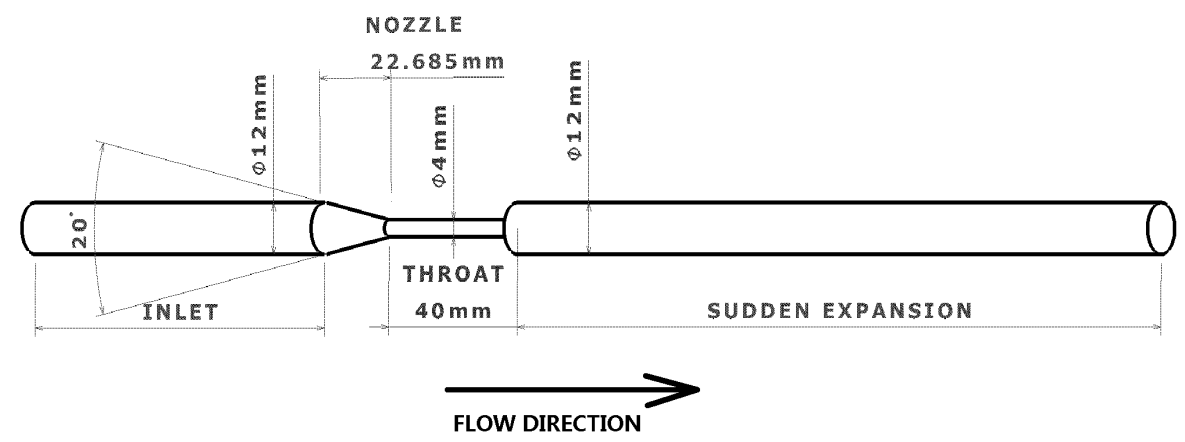

(a) Geometry of the nozzle

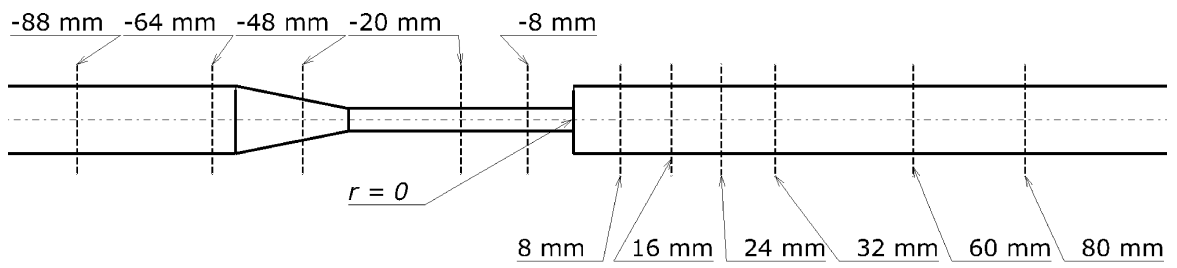

Axial position of cross-sectional cuts

(b) FDA defined locations of cross-sectional cuts for diagnostics

Figure 1: FDA medical nozzle specifications

This axisymmetric geometry consists of an inlet section of diameter $D=12 \mathrm{~mm}$, a $10^{\circ}$ half-conical convergent nozzle, a throat section of diameter $d=4 \mathrm{~mm}$ and length $l=10 \mathrm{~d}$ and a $D=12$ mm sudden expansion section at the end. Lengths of inlet and sudden-expansion sections are left to be determined by CFD research teams. However, these lengths in FDA experiments were significantly long in order to fully stabilize the flow and minimize the influence of any potential perturbations before the nozzle entrance. The following experimental range of laminar to fully turbulent throat Reynolds numbers $\left(R e_{t h}\right)$ has been tested by FDA [5]: $R e_{t h}=500,2000,3500$, 5000 and 6500 . Velocity and shear stress data have been made available for all these flow conditions but turbulence measurements in the flow domain or at the inlet were not reported.

Despite its relatively simple geometry, the proposed FDA benchmark case is a very difficult configuration both for an accurate experimental investigation and CFD prediction. The flow accelerates through the convergent nozzle before entering into the throat featuring a sharp intersection between these two sections. At the throat exit, flow issues into the sudden expansion where it decelerates. When the $R e_{t h}$ is higher than 2000, flow is characterized by a breakdown to turbulence. In addition to sectional complex specificities, axisymmetric geometries have the known property of being increasingly unstable in tangential direction, particularly in the transitional Reynolds number range [6, 7].

As reported in [8], a number of CFD solvers and numerical approaches failed to produce relevant quantitative data. The FDA interlaboratory effort gathered 28 different CFD groups over the world, which mainly based their blinded CFD investigations on Reynolds-Averaged NavierStokes (RANS) numerical methods. While a large number of them reported satisfactory results in the laminar case $\left(R e_{t h}=500\right)$, only two of them were able to match experimental centreline 
velocity at one of higher $R e_{t h}$ numbers. This FDA's CFD interlaboratory report [3, 8] practically suggested a need for different methods and numerical approaches.

This challenging FDA medical nozzle test case has then attracted a great amount of attention in the computational biomedical community, in particular as a validation test case for CFD. Different numerical methods were tested: a variant of direct numerical simulation (DNS) method [9], the lattice Boltzmann method [10], large-eddy simulation (LES) [11, 12], unsteady RANS (uRANS) or hybrid RANS/LES (HRL) approaches [13].

With the exception of the lattice Boltzmann method [10], which had difficulties to estimate meaningful velocity profiles in this complex test case and exhibited a large dependency on the lattices orientation, all other works reported flow averages in good agreement with the experimental quantitative data. DNS simulations performed on the open platform Life $V$ in [9] yielded surprisingly good results with coarse grids. However, for some transitional Reynolds number values, results were unstable and reported to depend on the numerical scheme and on the size of the computational domain. These initial results were then improved by careful mesh adaptation and careful time-step selection [9].

The commercial ANSYS ${ }^{\circledR}$ FLUENT_14 CFD solver was used in investigations reported by $[12,13]$. The domain was discretized using the structured O-grid and hexagonal mesh elements with strong refinement towards the walls and the critical cross-sections. Janiga [12] analyzed only the fully turbulent case $\left(R e_{t h}=6500\right)$, imposing a laminar parabolic inlet, even though the inlet flow was mildly turbulent $(R e=2167)$ as reported in the experiments [5]. Reportedly, velocity statistical data using the Smagorinsky model in Fluent were in very good agreement in the sudden expansion region and slightly underpredicted in the throat. uRANS and HRL methods reported in [13] yielded a variable performance. While the Fluent detached eddy simulations (DES) failed to correctly predict the $R e_{t h}=3500$ case, SST uRANS overpredicted turbulence levels in the same case. The dynamic HRL model used within the FLUENT_14 solver yielded satisfactory results. In the validation study of transition-sensitive uRANS, turbulence-injection was the method requirement in Fluent and the procedure was verified on a capillary tube test case, as denoted in [13].

Another study based on LES with the immersed boundary method [11] within the in-house WenoHemo code incorporated the WENO finite-difference scheme and a version of the transition sensitive Vreman's subgrid scale (SGS) model [14]. Using fully structured Cartesian meshes and a very low Courant-Friedrich-Lewy (CFL) number $(C F L<0.1)$, results were slightly overpredicting turbulence for the $R e_{t h}=3500$ case and more largely in turbulent $R e_{t h}=5000$. In the follow-up of this work [15], the same group evaluated two contemporary SGS models against the classical Smagorinsky on an idealized stenotic flow model. In this work a counter-intuitive conclusion is drawn, endorsing the Smagorinsky model known for its many drawbacks, including too large dissipation and wrong near wall behavior, over the advanced SGS models like the so-called Vreman model [14] and $\sigma$-model [16].

\subsection{Transition in pipe flow type configurations}

One of the common ways to characterize the transition in a pipe flow, that is of a particular interest here, is by studying the response of the flow to small perturbations $[6,17,18,19,20]$. From the fluid mechanics point of view, the Poiseuille flow is linearly stable; therefore, the value of Reynolds number to inflict breakdown to turbulence should be infinite [21]. However, transition to turbulence is known to occur in the $R e=2000$ to 4000 range even in ideal test configurations. Small finite perturbations are expected to decay and be dissipated downstream or to be amplified in the case of transition to turbulence at higher Reynolds numbers. In the majority of prominent works on turbulence in pipe flow, turbulence first appears through the occurrence of hairpin structures in the flow as a consequence of disturbances at the entrance. They develop into small patches called puffs or eventually slugs [22]. Their growth, splitting and merging result in the generation of coherent structures and turbulence [18]. As elaborated in $[19,23]$ triggering the turbulence in a pipe shear flows largely depends on the inflow and 
experimental initial conditions. Effects at the inlet are then related to the occurrence of transition via three-dimensional structures as traveling waves [7, 24]. There is actually a critical amplitude of inlet perturbations that will have enough life time before total decay, albeit without generating turbulence. Small inlet perturbations with sufficient life time may thus be convected to regions where they may contribute to turbulence transition. In addition to propagation of vortical structures, near-wall disturbances may have a significant impact on the triggering of turbulence transition [20, 25]. As reported in [6], flow perturbations have a variable effect dependently on the location of the perturbations injection. Notably, a perturbation closer to the wall will eventually result in stretched lambda-vortex developing into a large-scale hairpin vortex. This effect is of significance in throats where undeveloped velocity profiles are present.

From this theoretical basis we can assume that small perturbations introduced at the inlet of the FDA's medical nozzle would be mostly dissipated downstream, but also that some of their traces might survive long enough to reach the higher velocity sections where they may be eventually amplified. In [6], a spatially developing DNS of a pipe flow is performed to simulate transition. Even for $R e=5300$ the flow with infinitesimal inlet perturbations goes back to laminar state, reaching developed turbulence for $R e>8000$. The perturbations at the inlet were in the range of $5 \%$ of turbulence intensity $(I \sim 5 \%)$. In another DNS study [18], direct perturbations were avoided, but an additional water jet was used to inflict disturbances of around $2.5 \%$.

Generally, the evolution of the imposed disturbances at the inlet depends on the configuration. Inlet perturbations with turbulence intensity as high as several tens of percents may be imposed in some cases without leading to transition. In some experimental studies inlet perturbations of up to $30 \%$ of turbulence intensity are reported without destabilizing the pipe flow. A commonly accepted value for minimally perturbed inlets in transition cases assessments as in [6, 18] is around $\sim 5 \%$.

\subsection{Purpose of the study}

Recently reported satisfactory results of numerical simulations in the case of the FDA idealized medical nozzle announce a promising perspective for LES in computational biomedical engineering. However, a number of important aspects have been overlooked in these works. In particular, the robustness of the numerical prediction is not properly assessed. This represents an issue for the consistent and reliable use of CFD tools [3, 8]. With the motivation to properly assess the LES abilities in this FDA biomedical device test-case, LES are performed with an in-house CFD code $[26,27]$. In the current investigation special attention is paid to avoid adaptation of the numerical parameters from a priori knowledge of the experimental results, in a way to mimic the blinded study conditions. The influences of different numerical parameters on the results are independently analysed. The robustness of the numerical results is then investigated with or without inlet perturbations.

\section{Methodology and numerical environment}

Large-eddy simulation has emerged in the recent decade as one of the most powerful methods in CFD research. LES provides an attractive compromise between the costly DNS and the RANS methods which lack predictive capability. With the current and increasing computating power, LES potentially represents an excellent tool for medical devices design and applications, medical practice and personalized medical treatments when blood flow transitions to turbulence. In the past decades, LES has evolved rapidly with the advances in subgrid scale modeling, wall modeling and inclusion of immersed boundary methods (IBM), as for example reviewed in [28].

In the current investigation, the so-called YALES2BIO solver (www.math.univ-montp2.fr/ $\sim$ yales2bio) was used. YALES2BIO is an in-house high-fidelity CFD tool [27] developed at IMAG (Montpellier, France) in cooperation with CORIA from Rouen, France [29]. YALES2BIO is developed from YALES2 (www.coria-cfd.fr/index.php/YALES2), dedicated to LES and DNS of incompressible reactive flows. YALES2BIO includes an LES incompressible solver, an 
IBM solver for capsules in hemodynamics research [26], Arbitrary-Lagrangian-Eulerian (ALE) framework [30] and LES/IBM coupled with the LMGC90 [31] finite element solver for fluidstructure interaction (FSI) simulations of blood vessels and artificial organs [32].

\subsection{Numerical environment}

The filtered continuity and Navier-Stokes equations for a Newtonian and incompressible fluid read:

$$
\begin{gathered}
\frac{\partial \bar{U}_{i}}{\partial x_{i}}=0 \\
\frac{\partial \bar{U}_{i}}{\partial t}+\frac{\partial \bar{U}_{i} \bar{U}_{i}}{\partial x_{j}}=-\frac{1}{\rho} \frac{\partial \bar{P}}{\partial x_{i}}+\nu \frac{\partial^{2} \bar{U}_{i}}{\partial x_{j} \partial x_{j}}-\frac{\partial \tau_{i j}}{\partial x_{j}}
\end{gathered}
$$

where, $\nu$ is the kinematic viscosity, $\rho$ the density, $U_{i}$ the velocity components and $P$ the pressure. Overlined symbols denote the filtered quantities fluctuating over the scales that are large enough to be directly resolved. The residual stress tensor $\tau_{i j}={\overline{U_{i} U_{j}}}_{-} \bar{U}_{i} \bar{U}_{j}$ accounts for the unresolved subgrid scale effects and is modelled as:

$$
\tau_{i j}=-2 \nu_{S G S} \bar{S}_{i j}
$$

with $\bar{S}_{i j}=\frac{1}{2}\left(\frac{\partial \bar{U}_{i}}{\partial x_{j}}+\frac{\partial \bar{U}_{j}}{\partial x_{i}}\right)$ representing the filtered rate-of-strain tensor and $\nu_{S G S}$ representing the SGS model specific eddy viscosity. The so-called $\sigma$-model [16] is used, where $\nu_{S G S}$ reads:

$$
\nu_{S G S}=\left(C_{\sigma} \Delta\right)^{2} \mathcal{D}_{\sigma}(u)
$$

where $C_{\sigma}=1.35$ is the SGS model constant, $\Delta$ is the subgrid characteristic length scale and $\mathcal{D}_{\sigma}$ is a differential operator associated with the model, which reads:

$$
\mathcal{D}_{\sigma}(u)=\frac{\sigma_{3}\left(\sigma_{1}-\sigma_{2}\right)\left(\sigma_{2}-\sigma_{3}\right)}{\sigma_{1}^{2}}
$$

with $\sigma_{1} \geq \sigma_{2} \geq \sigma_{3} \geq 0$ representing the three singular values of the local velocity gradient tensor. This model was selected because of its capability to produce zero SGS viscosity in laminar flows; its effectiveness was also demonstrated recently in the case of an unsteady jet-wall interaction configuration [33]

YALES2BIO operates on fully unstructured numerical grids consisting of tetrahedra, prisms, pyramids or hexahedra. The fluid equations are discretized on an Eulerian fixed grid with a 4th-order finite-volume scheme. The projection method introduced by Chorin [34] is used to solve the incompressible Navier-Stokes equations. The algorithm consists of a prediction step where time-advancement is performed with a central 4th-order time scheme.

In order to assess the influence of numerics, two time-advancement integration schemes were considered. The first one was an explicit low-storage Runge-Kutta scheme of the 4th order, RK4 [35]; the second was an advanced 4th-order accurate time scheme, TFV4A [36], which features an adjustable built-in diffusion. This scheme is a linear combination of the RK4 scheme and the TTG4A scheme [37]. While the first scheme is not dissipative, the second introduces numerical diffusion, which aims at damping the dispersion errors inherent to the central spatial schemes. The blending of the two schemes is fixed in the present paper to $80 \%$ of the RK scheme and $20 \%$ of the TTG4A scheme in order to minimize the numerical diffusion.

To correct the predicted velocity, a divergence-free velocity is evaluated at each time-step by solving a Poisson equation via the Deflated Preconditioned Conjugate Gradient (DPCG) algorithm, as detailed in [38].

\subsection{Test model}

From the original FDA case, a single model geometry is adopted with substantially long inlet $\left(12 D_{\text {inlet }}\right)$ and outlet $\left(17 D_{\text {inlet }}\right)$ sections to avoid any boundary effects. The same computational 


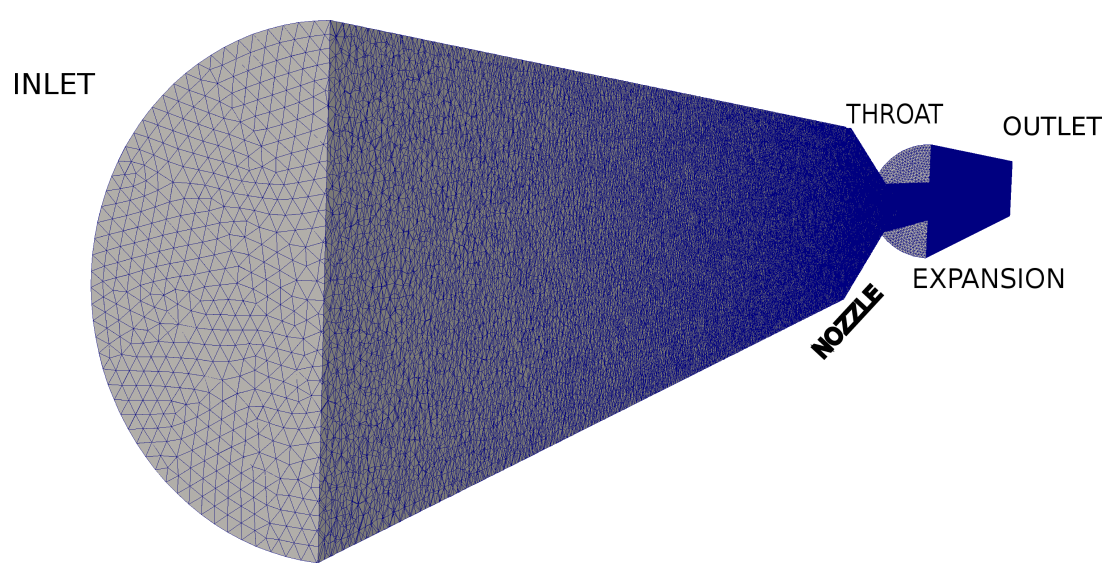

Figure 2: Split-view of meshed FDA idealized medical nozzle

domain was used in all computations. The geometry is axisymmetric, with the axis aligned with the $x$ direction, which is the streamwise direction. In terms of discretization of the computational domain, our preliminary tests showed that results were very sensitive to local grid refinement. With the intention not to presume the resulting flow field in any way, a fully unstructured grid with tetrahedral elements is constructed with almost no local mesh refinement in the domain. Notably, no mesh stretching was used near the solid boundaries; the flow being laminar in the nozzle and the throat, the number of elements per diameter used in the different mesh resolutions (see entry $(D / h)_{t h}$ in table I) is enough to resolve the near-wall gradients. Note that at the end of the sudden expansion region, the boundary layer thickness is approximately one fourth of the diameter. At the sudden expansion, the number of elements in the laminar shear layer thickness for coarse, medium and fine grids is thus 3, 5 and 8, respectively. In addition, avoiding the use of local refinement has the advantage of simplifying the grid description for further comparisons. Note that tetrahedral grids were preferred, as they are more generally adapted to complex configurations. Additional tests (not presented) suggest that the conclusions of the paper are also valid for hexahedral meshes. An example of grid is depicted in Figure 2. All grids were generated using the ANSYS ${ }^{\circledR}$ GAMBIT meshing software. A very high grid quality in terms of aspect ratio and equiangular skewness is achieved by specifying the number of mesh improvement iteration steps, maximal skewness and angle in Gambit solver mesh merge operations. All mesh elements were below $0.4-0.5$ value of equiangular-skew with $70 \%$ of them in the range 0 to 0.2 (0being best, 1-worst). The resulting grids were only slightly stretched toward the inlet and outlet

Table 1: Main characteristics of the three meshes used in the study: the number of cells, average, minimum and maximum cell size are provided, together with the number of elements in the throat diameter $(\mathrm{D} / \mathrm{h})_{t h}$. In addition, maximum values of the SGS-to-laminar viscosity ratio are reported.

\begin{tabular}{lllllll}
\hline \hline Mesh & No. cells & $\begin{array}{l}\text { cell } h_{\text {avg }} \\
{[\mathrm{m}]}\end{array}$ & $\begin{array}{l}\text { cell } h_{\min } \\
{[\mathrm{m}]}\end{array}$ & $\begin{array}{l}\text { cell } h_{\max } \\
{[\mathrm{m}]}\end{array}$ & $\begin{array}{l}(\mathrm{D} / \mathrm{h})_{t h} \\
{[\text { elements }]}\end{array}$ & $\left(\nu_{S G S} / \nu\right)_{\max }$ \\
\hline Coarse & $5 \times 10^{6}$ & $3.4 \times 10^{-4}$ & $3.2 \times 10^{-4}$ & $3.6 \times 10^{-4}$ & 13 & 3.324 \\
Medium & $15 \times 10^{6}$ & $2.0 \times 10^{-4}$ & $1.9 \times 10^{-4}$ & $2.3 \times 10^{-4}$ & 21 & 2.535 \\
Fine & $50 \times 10^{6}$ & $1.4 \times 10^{-4}$ & $1.2 \times 10^{-4}$ & $1.7 \times 10^{-4}$ & 34 & 1.194 \\
\hline \hline
\end{tabular}

boundaries. Three grids were considered: the coarse grid contains 5 million tetrahedral elements, the medium grid 15 million and the fine grid 50 million. Mesh cell sizes in the sudden expansion region are tabulated in Table 1 . The ratio shown in the last column of Table 1 represents the maximal value of LES model viscosity $\nu_{S G S}$ scaled by the fluid kinematic viscosity $\nu$. This ratio is a measure of the contribution of the subgrid model for each grid level. It shows that the DNS 
regime (for which $\nu_{S G S}<<\nu$ is expected) is not reached, even in the case of the fine mesh.

\subsection{Flow Conditions and Simulation Details}

The properties of the blood analogue fluid, with assumed Newtonian nature, were specified by the FDA inter-laboratory test-conditions: the dynamic viscosity is $\mu=3.5 \times 10^{-3} \mathrm{~Pa} \cdot \mathrm{s}$ and the density is $\rho=1056 \mathrm{~kg} / \mathrm{m}^{3}$. As all cases presented in this paper are for $R e_{t h}=3500$ and lower, a laminar Hagen-Poiseuille flow profile is imposed at the inlet:

$$
\bar{u}(r)=2 \cdot \bar{u}_{\text {mean }}\left(1-\frac{r^{2}}{\left(D_{\text {inlet }} / 2\right)^{2}}\right)
$$

where $\bar{u}_{\text {mean }}$ is the mean inlet velocity and $r$ the radial location at the inlet boundary.

A convective boundary condition is imposed at the downstream end:

$$
\frac{\partial \phi}{\partial t}+u_{c} \frac{\partial \phi}{\partial x_{n}}=0
$$

where, $\phi$ is any scalar value or velocity component, $u_{c}$ the convective velocity and $x_{n}$ is the coordinate in the direction of the outward normal at the boundary. Thus, the convective outlet condition neglects the diffusive effects and assumes the flow as purely advective near the boundary.

In terms of solid walls, Hariharan et al. [5] report a typical roughness value of 0.5 microns in FDA's medical device model. Wall boundary conditions were thus specified to be non-slipping smooth walls.

Time step in the calculations was governed by the Courant-Friedrichs-Lewy (CFL) condition which in the current investigation ranges from $\mathrm{CFL}=0.1$ to $\mathrm{CFL}=0.9$ as a part of assessment of the effect of the numerics on the flow field.

In the evaluation of numerical robustness, a turbulence injection (TI) type of perturbation was imposed at the nozzle inlet. The so-called turbulence injection actually represents an inhomogeneous turbulent noise superimposed over the original initial velocity profile via box-type adjacent zone at the inlet. No attempt was made to inject coherent turbulent structures. Thus, the procedure only generates small velocity fluctuations at the inflow. This perturbation boundary condition was specified as a turbulence intensity percentage of the mean velocity at the inlet with a turbulence length scale defined as a $3.8 \%$ of inlet diameter. Inlet TI from 0.5 to $5 \%$ of turbulence intensity was tested.

With a number of numerical and flow parameters analysed, the study was mainly concentrated on the $R e_{t h}=3500$ case, which provides a flow field at the verge of transition with anticipated turbulence breakdown in the sudden expansion. $R e_{t h}=3500$ in the throat yields $R e_{i n}=1167$ at inlet with the resulting mean velocity of $\bar{u}_{i n}=0.3223 \mathrm{~m} \cdot \mathrm{s}^{-1}$.

Table 2: Main characteristics of the simulations performed. Note that some of the rows correspond to a family of runs (e.g. Run 01 was done for two Reynolds number values, everything else being unchanged).

\begin{tabular}{llllll}
\hline \hline Run \# & $\mathrm{Re}_{t h}$ & CFL & Grid & Scheme & Turbulence inj. \\
\hline Run 01 & 500,3500 & 0.6 & $15 \mathrm{M}$ & TFV4A & $0 \%, 3 \%$ \\
Run 02 & 3500 & 0.6 & $50 \mathrm{M}$ & TFV4A & $0 \%$ \\
Run 03 & 3500 & $0.2,0.6,0.8$ & $5 \mathrm{M}$ & RK4, TFV4A & $0 \%$ \\
Run 04 & 3500 & $0.1,0.2,0.6,0.8$ & $15 \mathrm{M}$ & RK4, TFV4A & $0 \%$ \\
Run 05 & 3500 & 0.6 & $5 \mathrm{M}, 15 \mathrm{M}, 50 \mathrm{M}$ & TFV4A & $0 \%$ \\
Run 06 & 3500 & 0.6 & $15 \mathrm{M}$ & RK4, TFV4A & $3 \%$ \\
Run 07 & 3500 & 0.6 & $15 \mathrm{M}$ & TFV4A & $0.5 \%, 1 . \%, 3 \%, 5 \%$ \\
Run 08 & 3500 & 0.6 & $15 \mathrm{M}, 50 \mathrm{M}$ & RK4, TFV4A & $3 \%$ \\
Run 09 & 3500 & $0.2,0.6$ & $15 \mathrm{M}$ & TFV4A & $0 \%, 3 \%$ \\
Run 10 & 3500 & $0.2,0.6$ & $15 \mathrm{M}$ & RK4 & $0 \%, 3 \%$ \\
\hline \hline
\end{tabular}


All the test cases were first run for a long physical time to settle the flow. For $R e_{t h}=3500$ cases, the flow initialization time was $t_{i}=2 \mathrm{~s}$. This corresponds to more than 50 convective time units considering the sudden expansion diameter as the length scale. Gathering of flow statistics was performed during $t_{s}=3 \mathrm{~s}$. Table 2 details the numerical and physical parameters for the series of calculations performed for this study.

\section{Results Discussion}

Numerical results of the flow in the FDA's ideal medical device are reported in order to highlight the conditions affecting the flow field. One of the merits of LES is to directly resolve the large turbulence structures in the flow, whereas the SGS model treats the effects of the subgrid structures. As such, LES should not need adaptations of geometry, mesh and turbulence models depending on the case, contrary to RANS approaches. However, influencing conditions, both physical and numerical, may have an important role that should be clarified.

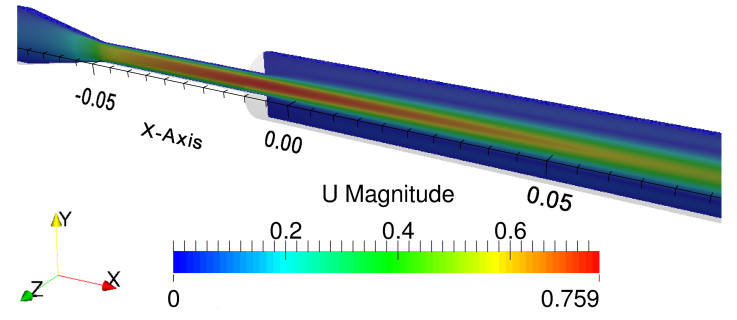

(a) Laminar $R e_{t h}=500$

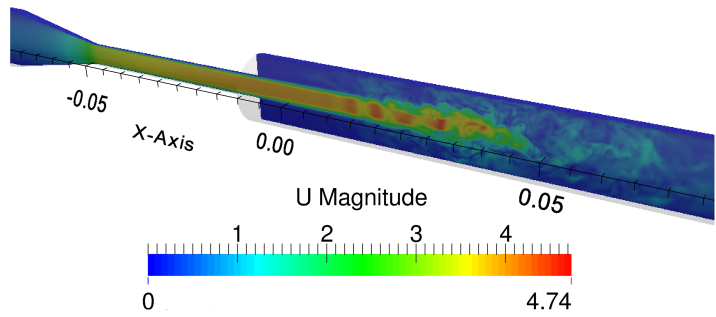

(b) Turbulent $R e_{t h}=3500$

Figure 3: Instantaneous velocity fields for two Reynolds number cases - Run 01

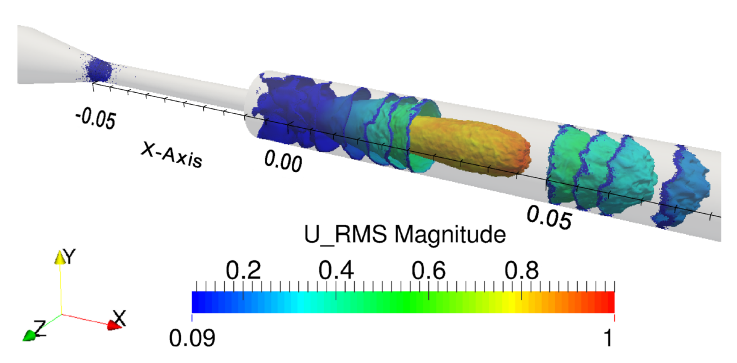

(a) No turbulence injection

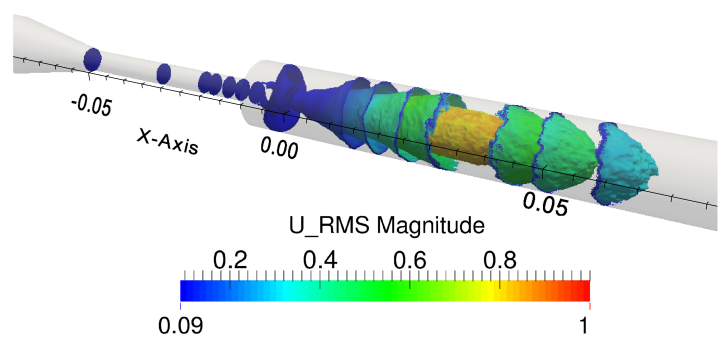

(b) $3 \%$ turbulence-injection

Figure 4: Iso-surfaces of pressure coloured by the amplitude of the RMS velocity. Left: Run 01 at $R e_{t h}=3500$. Right: Run 06 with TFV4A.

Instantaneous velocity fields in Figure 3 depicts two $R e_{t h}$ cases, a laminar and a turbulent case. In the low-speed case at $R e_{t h}=500$, shown in Figure 3(a), the flow remains laminar in the whole domain. YALES2BIO successfully predicts this laminar flow, without transition streaks or turbulence downstream of the sudden expansion. For the $R e_{t h}=3500$ case, the Reynolds number is first $R e=1166.67$ at the inlet. The flow then passes through the convergent nozzle to the throat with a $\delta=A_{i n} / A_{t h}=9$ inlet-to-throat aspect ratio. The flow at $R e_{t h}=3500$ in the throat then reaches the sudden expansion, where recirculation behind a plume and formation of effective mixing shear-layer occur, eventually leading to the jet breakdown to turbulence. The instantaneous velocity field in Figure 3 (b) shows that this flow structure is well recovered in the simulation. Considering the magnitude of the root-mean-square (RMS) velocity shown in Figure 4 (a), it can be deduced that growth of the disturbances occurs downstream of the 
sudden expansion with a peak at the jet breakdown location. RMS iso-surfaces are absent in the upstream fully laminar flow sections, except for dispersively distributed puffs concentrated near the wall at the throat entrance. Presumably, the sharp intersection between the convergent nozzle and cylindrical throat would inflict small disturbances close to the wall. In the case of turbulence injection (Figure $4 \mathrm{~b}$ ), where disturbances are introduced at inlet, traces of low RMS levels appear as a train of toroidal iso-surfaces along the throat. The effects of these small perturbations are further detailed in the results sections.

\subsection{Streamwise flow field data distribution}

Quantitative data of the resulting flow fields are extracted and compared with FDA experimental data. Presented data were obtained on the fine grid, a moderate $\mathrm{CFL}=0.6$ condition and TFV4A time scheme (Run 02). The resulting flow field is in excellent agreement with the FDA

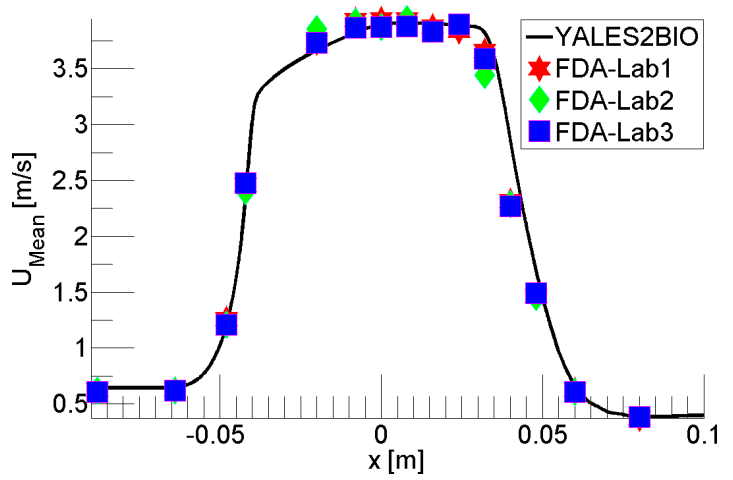

(a) Mean velocity at the axis

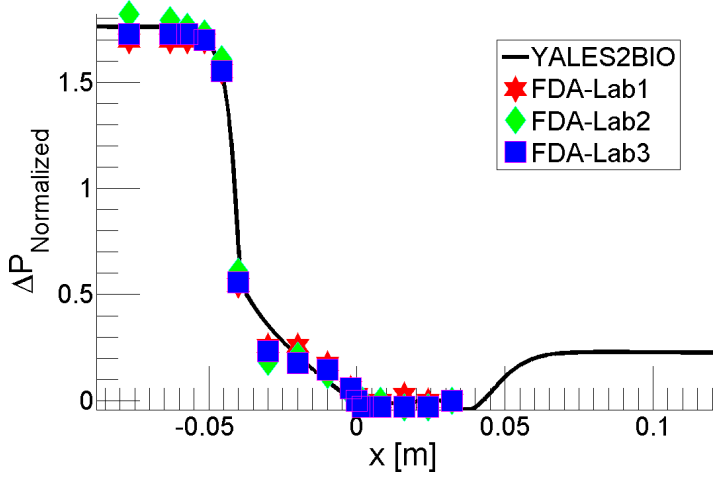

(b) Mean pressure along the walls

Figure 5: Streamwise evolution of mean flow quantities - Run 02

experimental data as depicted in Figure 5. The centerline velocity profile shown in Figure 5 (a) is in very good agreement, falling right in-between the three laboratories experimental results, both in the laminar and turbulent $(x \geq 0.04 \mathrm{~m})$ parts. Pressure data is presented in Figure $5(\mathrm{~b})$, as a pressure difference measured relative to the pressure at $\mathrm{x}=0\left(\Delta P=P-P_{x=0}\right)$, normalized by the dynamic pressure at the throat:

$$
\Delta P_{\text {Normalized }}=\frac{\Delta P}{\frac{1}{2} \rho \bar{u}_{t h}^{2}}
$$

A very satisfactory agreement is obtained between the numerical and experimental wall-pressure distribution. Pressure drop in the nozzle, pressure loss in the throat, plateau value in the sudden expansion and rise after the jet breakdown are all resolved with high accuracy.

\subsection{Effect of Numerics on the Jet Breakdown Prediction}

As shown in the previous section, the use of the fine grid with a moderate $\mathrm{CFL}=0.6$ and the TFV4A time scheme demonstrated an excellent performance to reproduce experimental data. However, alteration of any of these numerical parameters or their combination appears to affect the CFD outcome, which results in erroneous prediction of the breakdown location. For instance, for the medium grid and the TFV4A time scheme (Run 04), the flow field for two different CFL numbers are shown in Figure 6. A low CFL number predicts a much earlier turbulence transition than a higher CFL number. This trend is general when the TFV4A scheme is used and can be observed for the coarse and medium grids in details in Figure 7 (a) (c). On the contrary, cases with the RK4 time integration scheme in Figure 7 (b) appear more resilient to a change in CFL number. Nonetheless, Figure 7 (d) shows that in the medium grid cases with RK4, jet breakdown 


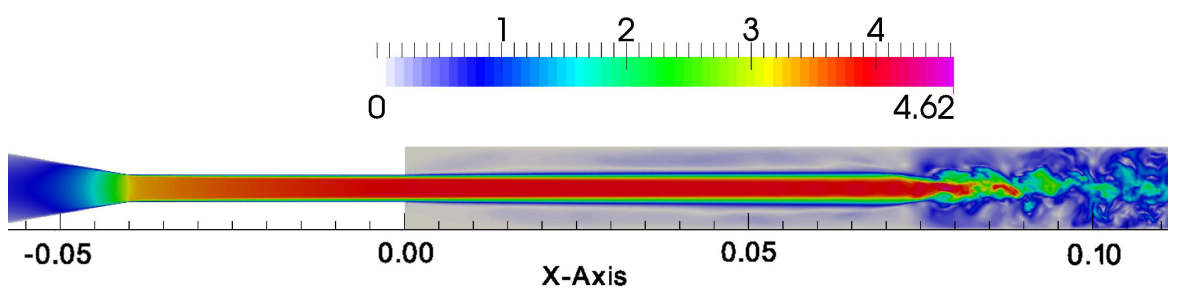

(a) $\mathrm{CFL}=0.6$

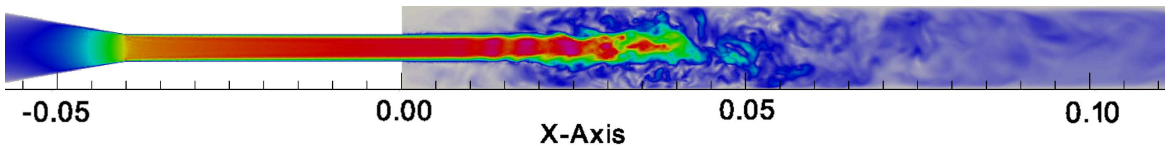

(b) $\mathrm{CFL}=0.1$

Figure 6: Instantaneous velocity fields from Run 04 (TFV4A) for two different values of the CFL

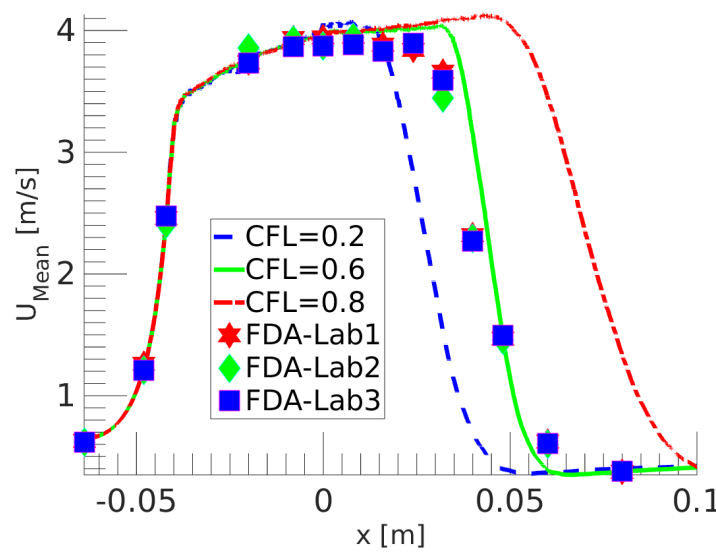

(a) 5M grid - TFV4A scheme

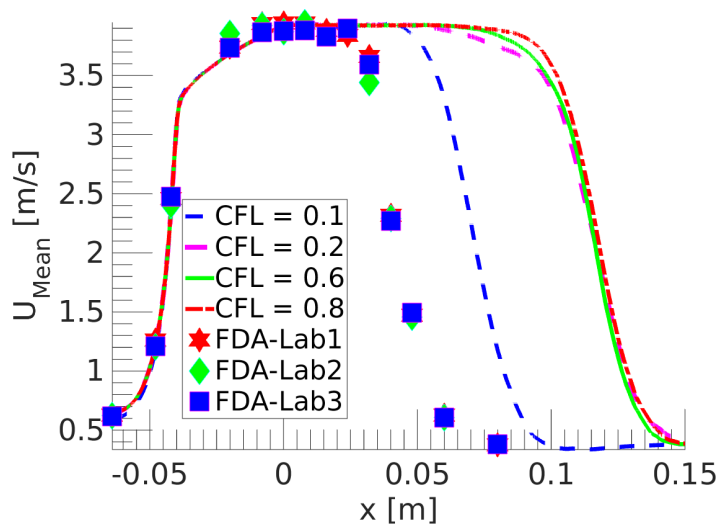

(c) 15M-grid - TFV4A scheme

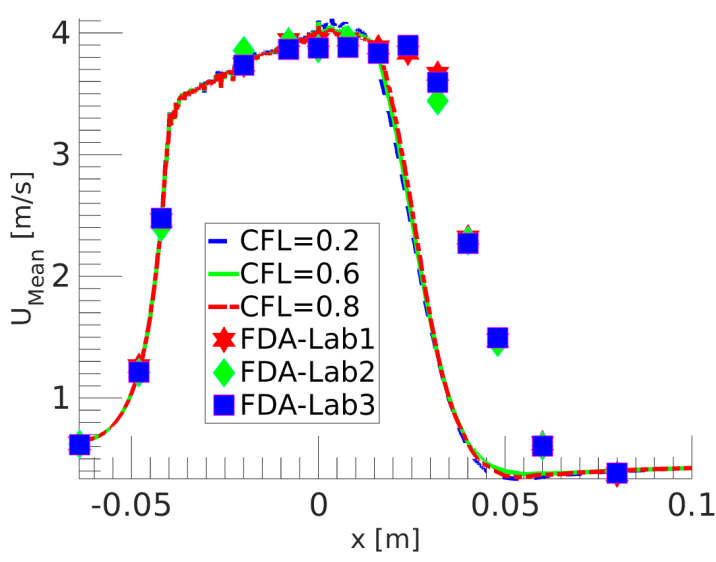

(b) 5M-grid - RK4 scheme

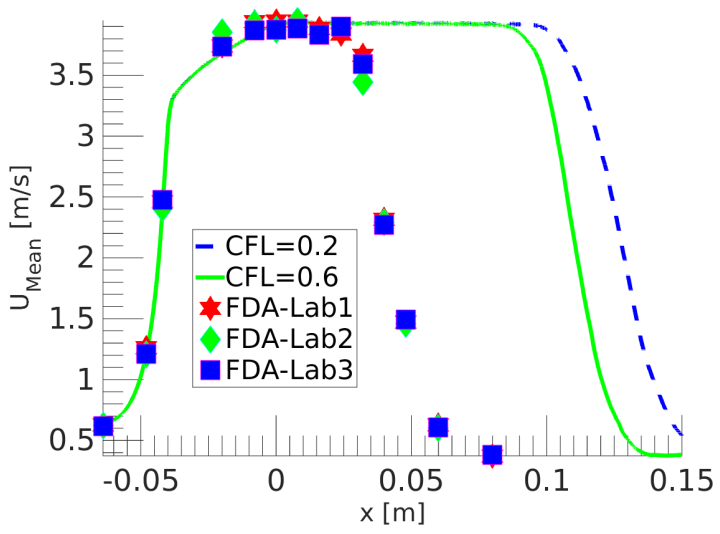

(d) 15M grid - RK4 scheme

Figure 7: Effect of the CFL number for a variety of numerical schemes and mesh resolutions. Axial profiles of the mean streamwise velocity. Top row: Run 03, 5M mesh ; Bottom row: Run 04, 15M mesh ; Left column: TFV4A scheme; Right column: RK4 scheme.

location is still sensitive to CFL number, results for $\mathrm{CFL}=0.2$ being worse than for $\mathrm{CFL}=0.6$. 
Inspecting the individual centreline profiles in Figure 7 can mislead the reader to the conclusion that coarse mesh with moderate $\mathrm{CFL}=0.6$ and TFV4A yields a fully satisfactory result; such a result is obtained here just by a chance, as suggested by the sensitivity of the results to numerical parameters. This illustrates how non-consistent and non-robust CFD simulation approach may obtain 'perfect' results in a very sensitive configuration. We note that our coarse

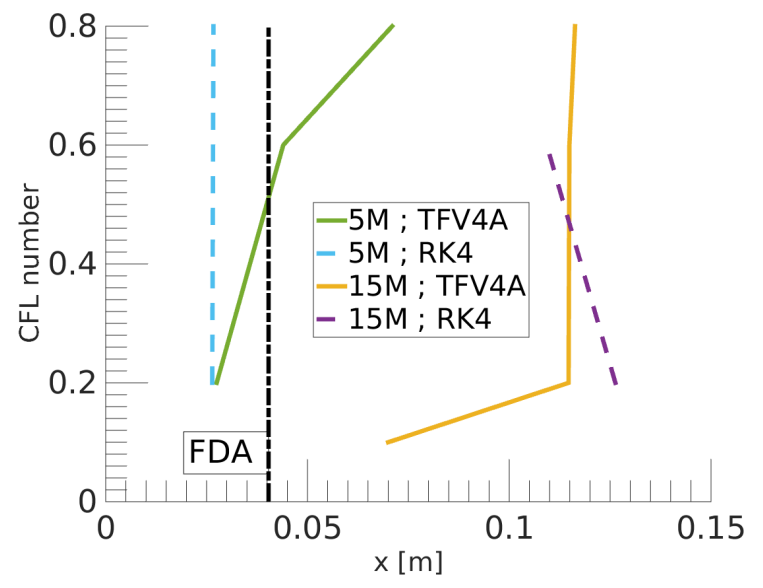

Figure 8: Effect of the CFL number on the centreline jet breakdown position for a variety of numerical schemes and mesh resolutions. Results from Runs 03 and 04.

mesh which is very similar to grid resolution reported in [11] reproduces the axial velocity profile with a slightly overestimated breakdown location using a low CFL number. This can be further altered by tweaking the time stepping, geometry and mesh as for example reported in [9]. In the

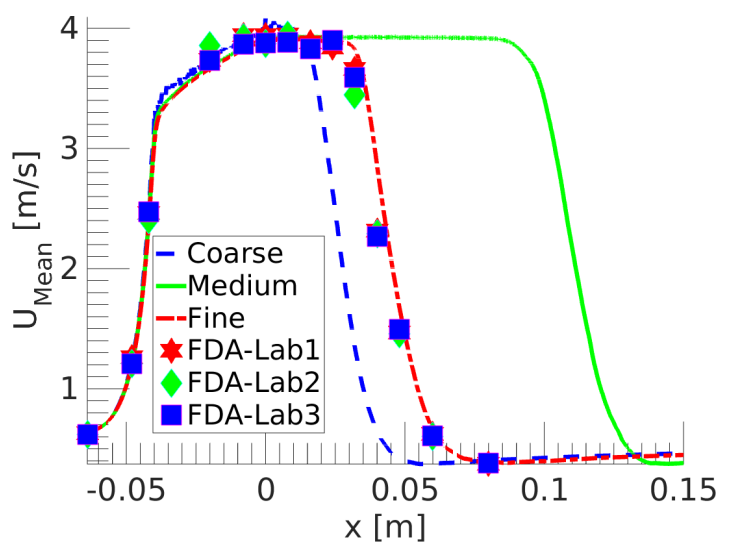

(a) centreline velocity

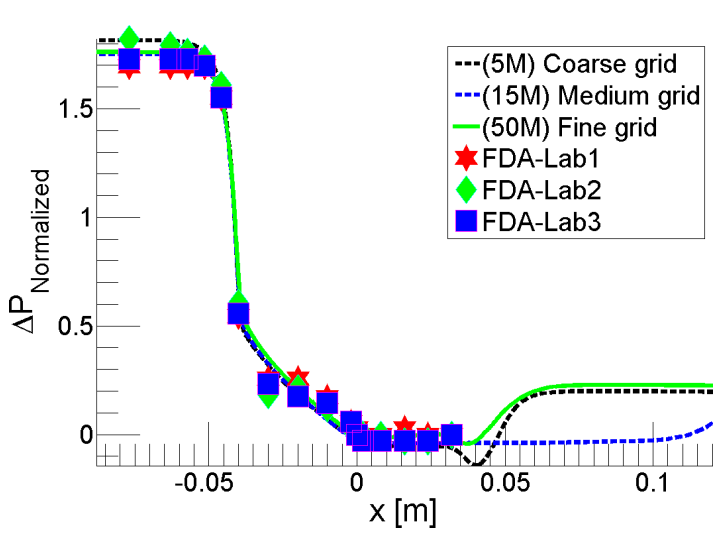

(b) Wall-pressure

Figure 9: Axial evolution of mean flow quantities for a variety of meshes at CFL=0.6 (Run 05).

current numerical study, the coarse grid is sufficient to reproduce the turbulence transition and breakdown with a moderately selected $\mathrm{CFL}=0.6$. When the mesh size is increased to 15 million elements, the jet breakdown occurs much farther using the two CFL values. In the case of $15 \mathrm{M}$ mesh, a very low CFL condition or time-step would be needed to match the experimentally reported turbulence breakdown.

The influence of numerics on the prediction of the axial location of the jet breakdown is summarized in Figure 8. Breakdown location is defined as the location of the inflection point on the axial profiles of mean axial velocity. Results are compared with experimental value of $x_{b}=0.04$ m. Figure 8 illustrates the shift of the jet breakdown with time step for different time schemes 


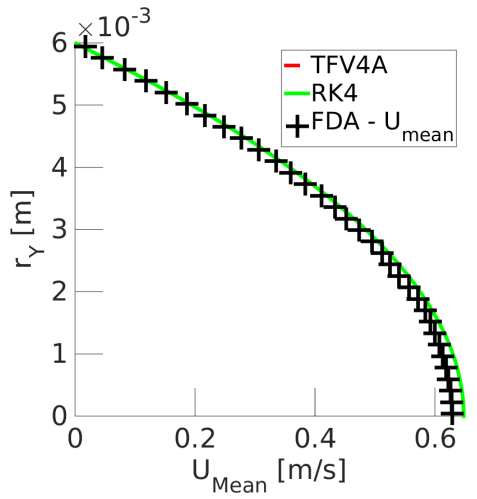

(a) $X=-0.064 \mathrm{~m}$

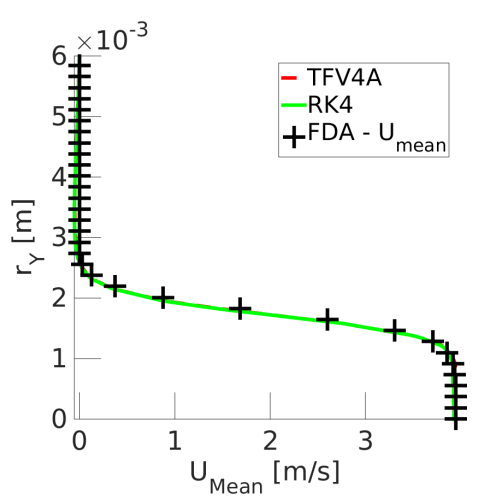

(d) $\mathrm{X}=0.008 \mathrm{~m}$

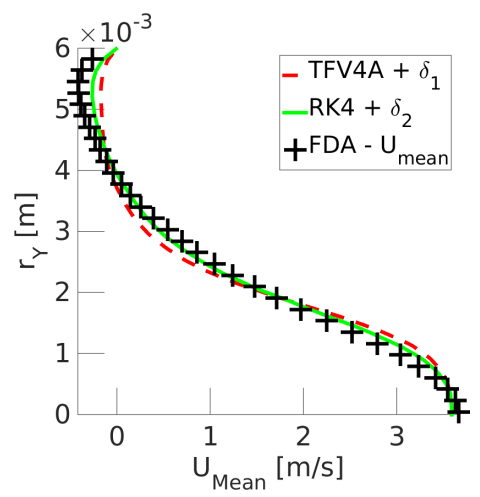

(g) $\mathrm{X}=0.032 \mathrm{~m}$

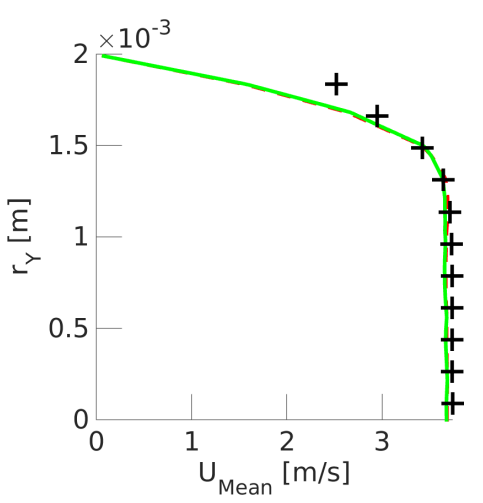

(b) $\mathrm{X}=-0.020 \mathrm{~m}$

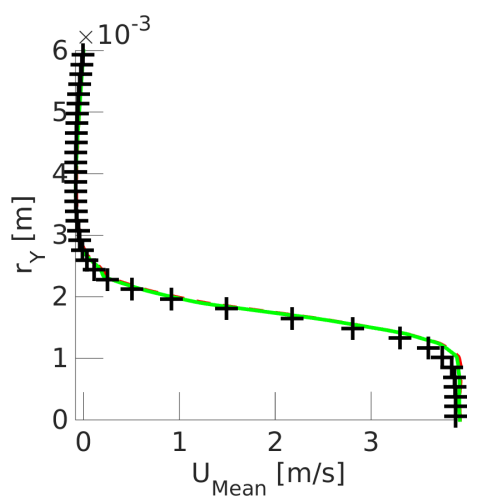

(e) $\mathrm{X}=0.016 \mathrm{~m}$

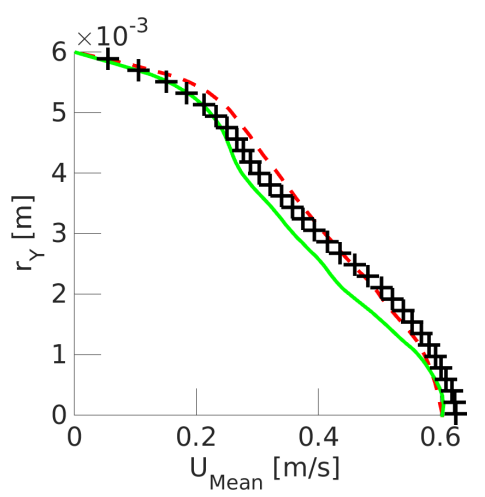

(h) $\mathrm{X}=0.060 \mathrm{~m}$

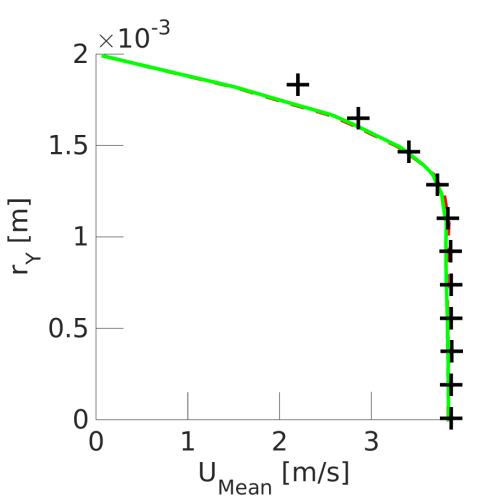

(c) $\mathrm{X}=-0.008 \mathrm{~m}$

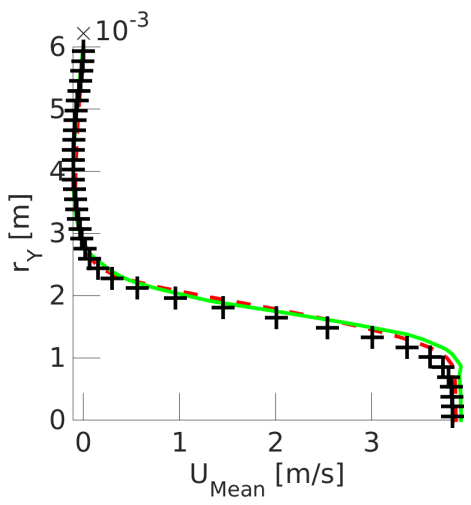

(f) $\mathrm{X}=0.024 \mathrm{~m}$

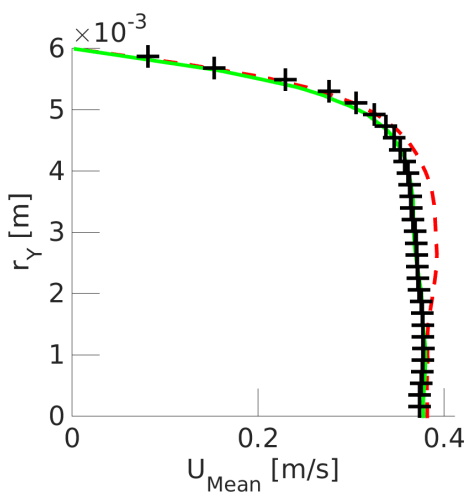

(i) $\mathrm{X}=0.080 \mathrm{~m}$

Figure 10: Profiles of mean streamwise velocity at various axial locations, without injection of turbulence. Results from Run 04 at $\mathrm{CFL}=0.6$.

and grids. This result further points out the potential influence of numerics on the CFD results in a case with turbulence transition.

Velocity and pressure axial profiles are displayed in Figure 9. They demonstrate the influence of grid resolution on the results. Pressure profiles appear correctly estimated in the regions upstream of the jet-breakdown. More downstream, differences are observed due to discrepancies in the jet breakdown location.

The axial location of a jet breakdown is extremely sensitive to numerical parameters. The purpose of the end of this section is to show that in spite of this sensitivity, flow structure is correctly predicted in the neighbourhood of the breakdown. Results for $\mathrm{CFL}=0.6$ and the medium 
grid are used. In order to further assess the quality of the simulations, radial velocity profiles are shown in Figure 10. Note that to perform a fair comparison, the position of the radial profiles should better be defined relatively to the jet breakdown position. The breakdown location is estimated at $x_{b}=0.04 \mathrm{~m}$ in the experiment, at $x_{b 1}=0.114$ for the TFV4A time scheme and $x_{b 2}=0.121$ for RK4. Therefore, the constant offset value for extraction axial location correction reads $\delta_{1}=0.074$ for TFV4A and $\delta_{2}=0.081$ for RK4 time scheme. This is a common practice in free jet computations as in [39], where jet breakdown location is known to be an extremely sensitive quantity.

Mean axial velocity profiles at inlet, nozzle and throat regions in Figure 10 (a, b, c) appear to be in very good agreement with the experiment. Only a minor overestimation of the shear co-flow is observed in Figure 10 (d), which probably comes from the experimental inability to perform measurements in region very close to the wall. In the breakdown zone in Figure 10 (g-i), axially offset profiles yield a satisfactory accordance with the experimental data. All radially distributed original velocity profiles before the breakdown incipient point and offset profiles afterwards match very nicely the experimental data and fit into the $95 \%$ certitude range. This comparison shows that even if the breakdown location is mispredicted, the radial flow structure is in excellent agreement with the experiments, which actually makes it a more robust assessment than turbulence transition location in the context of CFD validation.

As a conclusion, when high-quality grids are used, CFD yields satisfactory results before the sudden expansion region. In contrast, infinitesimal perturbations due to mesh, geometry and numerics can be amplified in the shear layer zone. Consequently, after the sudden expansion, the precise location for the transition to turbulence flow shows a striking lack of robustness.

\subsection{Perturbations at inlet}

Prediction of the jet breakdown location arises as the major issue in this FDA biomedical benchmark. Turbulence transition configurations are common in fluid mechanics and known to be excessively sensitive to any influence, which is even emphasized in axisymmetric geometries [39]. One of the prominent procedures in transition in pipe flow, as mentioned in the Introduction, is to perturb the inlet and observe the decay or the amplification of small disturbances. Turbulence intensity ( $\mathrm{I} \sim 0.5$ to $5 \%$ ) range is chosen for inlet perturbations in the current study and its effects on the LES prediction of transition are analysed. Note that in view of the experimental FDA data available in $[5,8,40]$ and private communication from their authors, this range guarantees that the injected fluctuations at inlet are significantly lower than the noise registered experimentally in inlet RMS velocity profiles. Figure 11 shows RMS and mean velocity radial profiles in log-scale at different cross-sectional cuts along the axial direction. Three calculations on the medium grid are compared: TFV4A without TI and with $3 \%$ TI at the inlet and RK4 with $3 \%$ TI. When available, the experimental data is also shown.

When inlet perturbations are injected, they rapidly dissipate before the throat. Between the inlet and the first experimental inlet sectional cut the RMS of velocity decreases by 2 orders of magnitude as seen in Figure 11 (a-b). The levels of RMS of axial velocity is typically 10 times smaller at the beginning of the converging section than at the inlet. This order of magnitude has been observed whatever the grid and numerical parameters used. Infinitesimal noise of $<10^{-7}$ range for the no TI case is supposedly scheme specific, most probably coming from DPCG pressure solver. RMS values for $\mathrm{TI}=0 \%$ case remain at this very low level, which is not visible in Figure 11 (b-e), in all upstream cuts before the sudden expansion. In the same cross-sections, the mean velocity profiles of the three cases considered perfectly match.

Close to throat entrance $(\mathrm{x}=-0.034 \mathrm{~m})$ in Figure $11(\mathrm{c})$, we observe a near-wall peak in the TFV4A cases emerging as the result of a sharp geometry change. This peak moves towards the axis and is being dissipated in the throat (Figure $11 \mathrm{~d}$-e). Full resolution of effects in the near-wall zone would require much higher mesh resolution or stronger wall adaptation at the throat entrance. We can assume that with noisier flows, as in experiments, enough disturbance generation at throat entrance may promote the development of some turbulence in the throat 


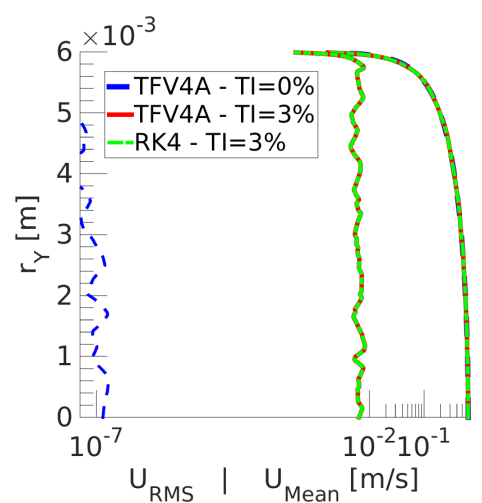

(a) $\mathrm{X}=-0.15 \mathrm{~m}$ (inlet)

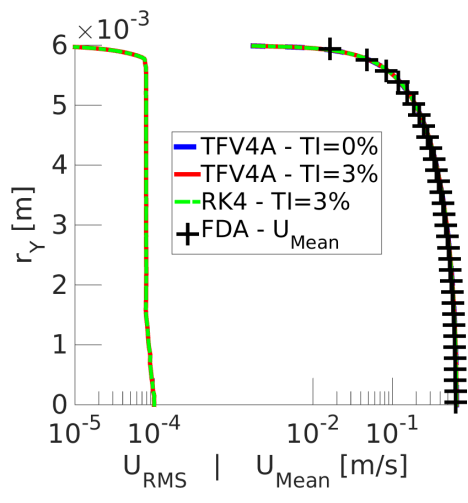

(b) $\mathrm{X}=-0.088 \mathrm{~m}$

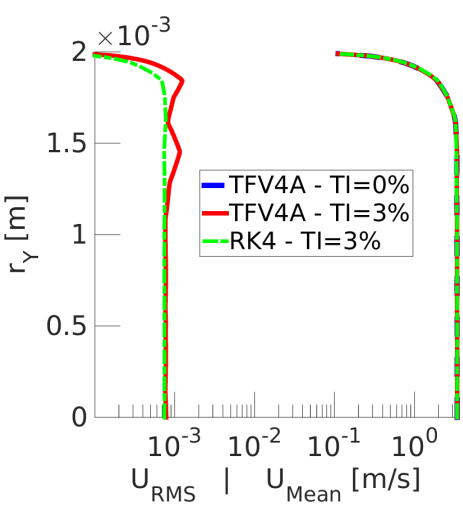

(c) $\mathrm{X}=-0.034 \mathrm{~m}$

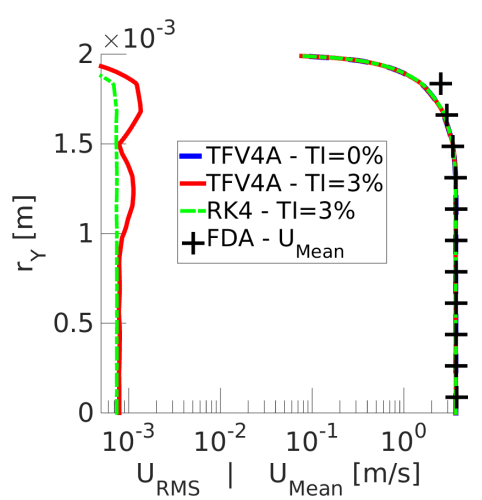

(d) $\mathrm{X}=-0.020 \mathrm{~m}$

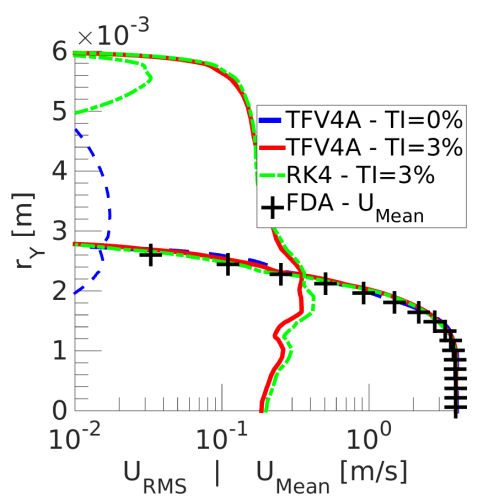

(g) $\mathrm{X}=0.016 \mathrm{~m}$

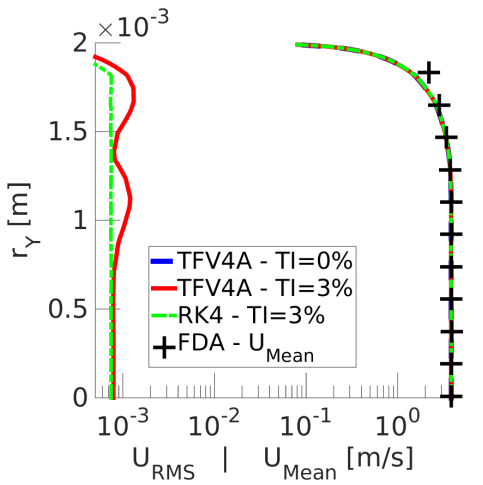

(e) $\mathrm{X}=-0.008 \mathrm{~m}$

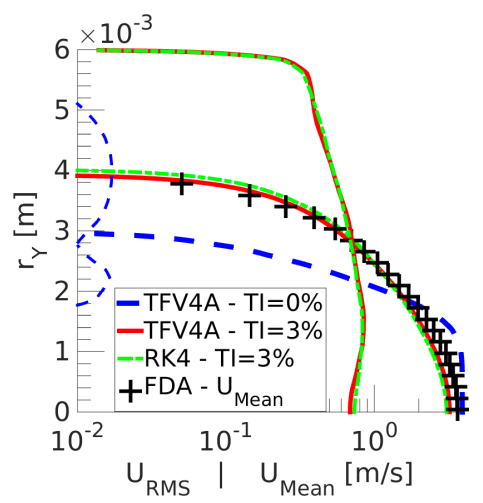

(h) $\mathrm{X}=0.032 \mathrm{~m}$

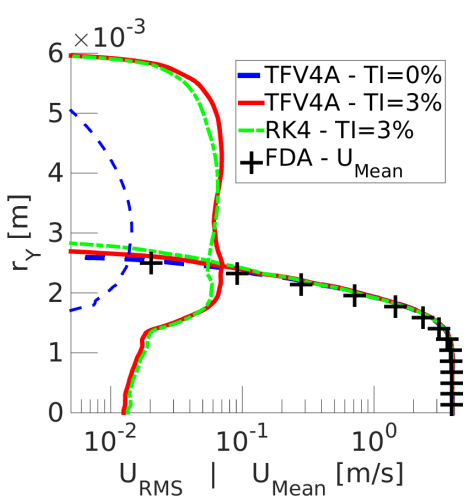

(f) $\mathrm{X}=0.008 \mathrm{~m}$

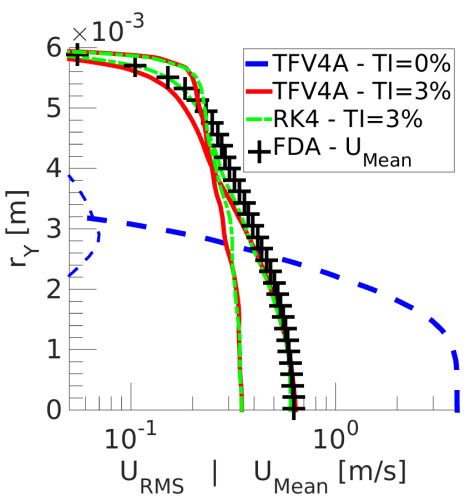

(i) $\mathrm{X}=0.060 \mathrm{~m}$

Figure 11: Profiles of streamwise mean and RMS velocity at various axial locations (semi log scale), without injection of turbulence. Results from Run 05 (medium grid) and Run 06.

boundary-layer with anticipated further effect on sudden-expansion jet.

Downstream of the sudden expansion, a mixing layer is formed between the outer zone and the jet plume, first associated with a peak in RMS profiles in Figure 11 (f). The values of RMS rapidly increase and spread over the whole section. This eventually leads to the jet-breakdown as seen in Figure 11 (h) where the RMS velocity peak, previously present in shear mixing layer region, moves to the centreline. Both time schemes with $3 \%$ inlet turbulence injection yield satisfactory agreement with the experimental data both in the transition and in the fully turbulent region (Figure 11 i). In the upstream cross-sections, mean velocity does not depend on TI: the three simulations displayed in Figure $11(\mathrm{a}-\mathrm{e})$ have exactly the same mean velocity profiles. However, 
even very small perturbations are significantly amplified in the sudden expansion region and both TI cases demonstrate a strong improvement in axial velocity predictions, as seen in Figure 11 $(\mathrm{g}-\mathrm{i})$.

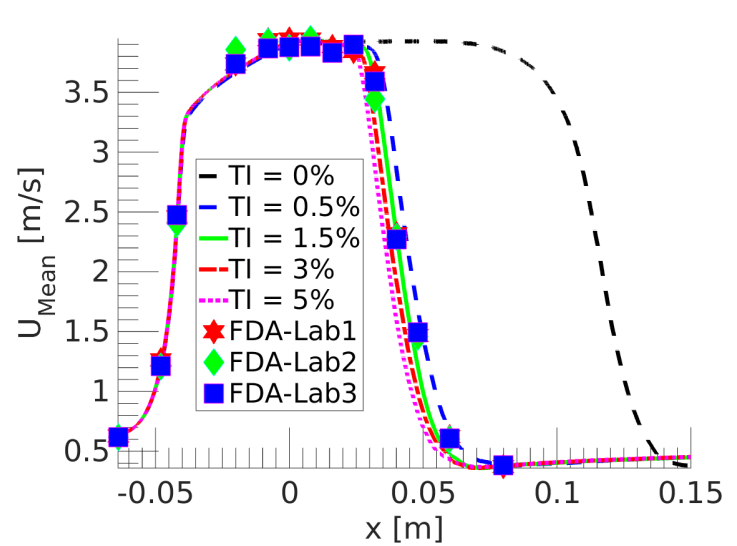

(a) TI levels at Medium (15M)-grid

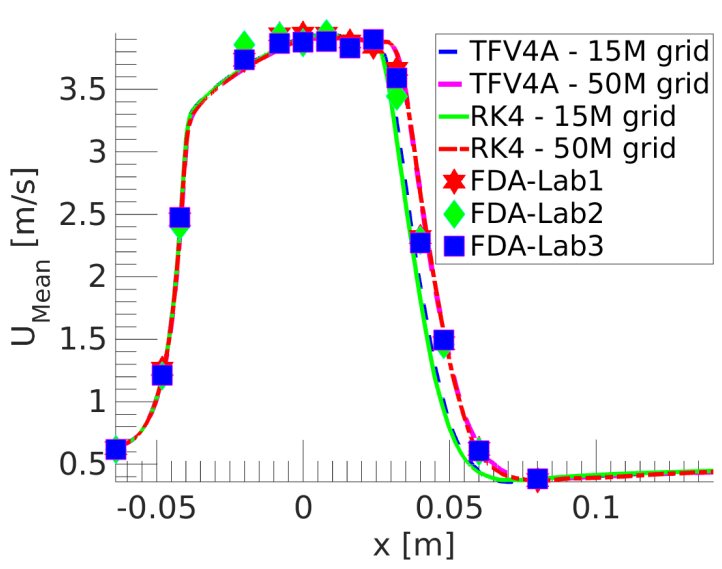

(b) $\mathrm{TI}=3 \%$ Mesh robustness

Figure 12: Effect of turbulence injection (left, Run 07) and numerical scheme/grid resolution (right, Run 08) on the axial evolution of mean streamwise velocity.

Centreline axial velocity profiles are displayed to show the influence of the small amplitude inlet perturbations on the flow. Figure 12 depicts the effects of the TI levels for the medium grid and the effect of grid resolution for $\mathrm{TI}=3 \%$ with the $\mathrm{CFL}=0.6$ condition. All cases with TI fall under the $95 \%$ certainty level and better. Additionally, all profiles with turbulence injection at the inlet converge to the experimental result whatever the discrepancies obtained in the cases without turbulence injection. When TI is used, the jet breakdown is notably independent of time scheme. All the cases with different turbulence injection levels are summarised in Table 3, where the location of the jet breakdown is compared to the experimental point at $x_{b}=0.040 \mathrm{~m}$.

Contrary to the cases without turbulence injection, these data show an asymptotic clustering

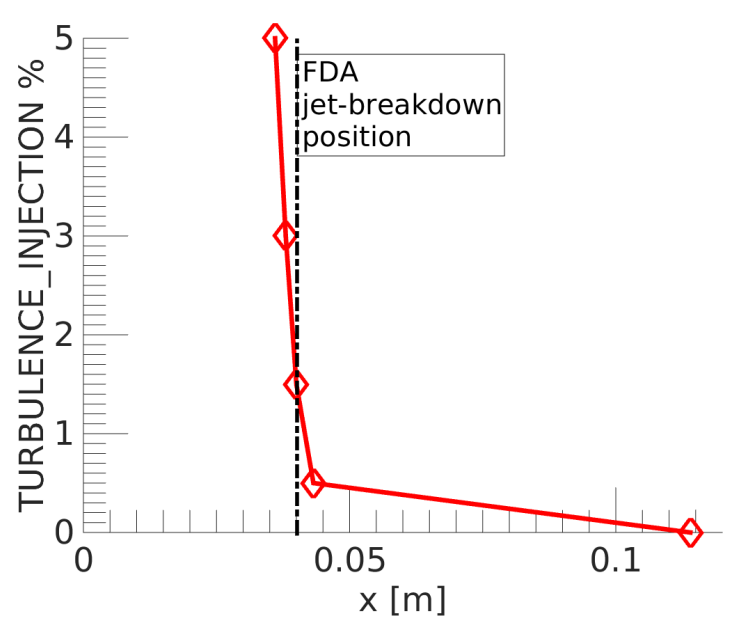

(a) Jet-breakdown location

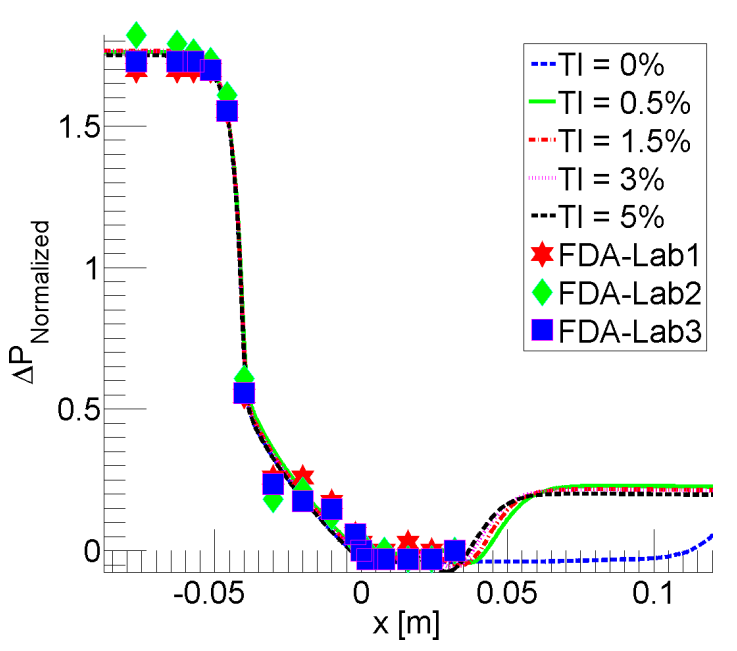

(b) Wall-pressure profiles

Figure 13: Effect of the intensity of turbulence injection on the location of jet breakdown (left) and axial evolution of wall pressure (right). Results from Run 07. 
Table 3: Jet breakdown vs. turbulence injection level for medium-coarse grid, TFV4A and $\mathrm{CFL}=0.6$

\begin{tabular}{lllllll}
\hline \hline Turbulence injection level & FDA & TI $=0 \%$ & TI=0.5\% & TI=1.5\% & TI=3.0\% & TI=5.0\% \\
Jet breakdown location $[\mathrm{m}]$ & 0.040 & 0.1141 & 0.0432 & 0.0401 & 0.0382 & 0.0366 \\
\hline \hline
\end{tabular}

of the jet-breakdown estimated locations around the experimental value as seen in Figure 13(a). Numerical pressure data shown in Figure 13 (b) appear to match the experimental data and are

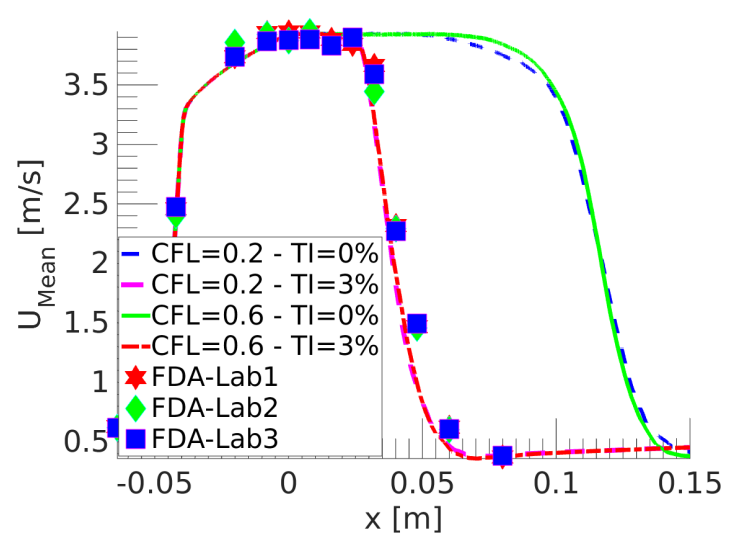

(a) TFV4A scheme, TI - CFL robustness

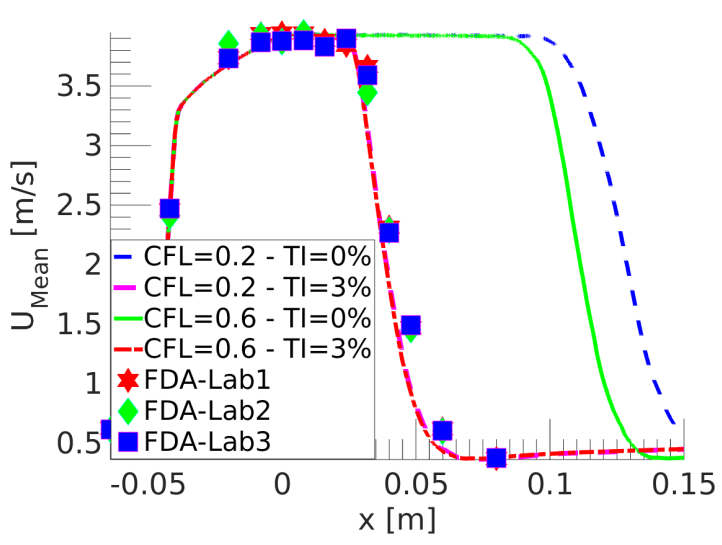

(b) RK4 scheme, TI - CFL robustness

Figure 14: Effects of turbulence injection and CFL number on the axial evolution of the mean streamwise velocity for the TFV4A (left, Run 09) and RK4 (right, Run 10) schemes.

not affected by the inlet perturbations upstream of the throat. Small differences in the pressure profiles, as anticipated, occur in the vicinity of the jet breakdown and are fully consistent with the centreline velocity profiles.

Finally, turbulence injection at the inlet also makes results robust to changes in the CFL number, as shown in Figure 14. Eventually, the LES results with turbulence injection are robust and consistent regardless of the employed time-scheme and CFL number. Results clearly demonstrate the effectiveness of the minimally inlet perturbation approach in obtaining a robust assessment of the location of turbulence transition, which is often overlooked in biomedical CFD studies.

\section{Conclusions}

The present study focused on the validation and robustness assessment of an LES approach in the framework of the challenging FDA medical nozzle benchmark. LES were performed with the in-house CFD solver YALESBIO. A fixed computational domain, with adaptation-free grids were considered. Three levels of tetrahedral grids, with 5, 15 and 50 million elements were used. The fine grid level was never reported in the literature to compute this case. No attempt was made to adapt the grid resolution or geometry of the nozzle, in order to mimic the blinded simulations. All grids and time-advancement schemes considered in this study performed satisfactory at inlet, nozzle and throat sections with very good agreement between CFD and experimental data. The major difficulty encountered in this investigation was to determine the exact location of the jet breakdown to turbulence downstream of the sudden expansion. Transition to turbulence of a jet plume in sudden expansion is found susceptible to a number of conditions of physical as well as numerical nature with downstream amplification of any upstream imperfections. Numerics (grid size, time advancement scheme, time step) showed a strong effect on the prediction of the jet breakdown location. 
The present results (and other numerical tests which were not presented) show that there is potentially a very large number of combinations of computational domains, grid resolution and stretching, space and time integration schemes and time step that would provide the same location of jet breakdown as in the experiment. However, these simulations obtained under the assumption that the upstream boundary condition is free of any disturbances turn out to be highly sensitive to any change in the numerical parameters. It is thus not that surprising that almost all blinded numerical predictions were unsatisfactory, while all subsequent numerical studies provide the same location of jet breakdown as in the published experimental data.

In the studies of turbulence transition, it is well known that the upstream noise is of extreme importance to be able to predict the exact location of the transition. Unfortunately, velocity fluctuations were not provided in the experimental data of the FDA test case. Any source of velocity disturbance coming from pumps, upstream section changes and/or bend may act as a source of small perturbations. In order to assess the effect of possible velocity fluctuations upstream of the test section, turbulence injection was introduced at the inflow of the domain. The levels of injected fluctuations were kept very small. In all cases, before the throat, RMS of velocity were more than 1000 times lower than the mean velocity; consistently, the amplitude of turbulence injection has a minor effect on the results. However, the presence of these fluctuations makes the jet breakdown prediction very robust to time scheme, CFL condition, as well as grid resolution. Finally, and more importantly, all cases with turbulence injection predict a jet breakdown location in very good agreement with the experiment. In the absence of physical velocity fluctuations, the flow is thus much more sensitive to numerical noise, which is influenced by any numerical parameter. Also, the robustness of the flow with regard to upstream velocity fluctuations probably explains why the experimental results from the database are consistent: the presence of small experimental noise, irrespective of its exact level, stabilizes the jet breakdown location.

Finally, this study illustrates the necessity to demonstrate the robustness of numerical results when dealing with blood flows, especially in the transitional regime. On top of preventing any predictive computation, any large sensitivity of the results to numerical parameters may be the print of a lack of understanding of the flow of interest.

\section{References}

[1] Marsden AL. Optimization in cardiovascular modeling. Annu. Rev. Fluid Mech 2014; 46:519-546, doi:10.1146/annurev-fluid-010313-141341.

[2] Marsden AL, Bazilevs Y, Long CC, Behr M. Recent advances in computational methodology for simulation of mechanical circulatory assist devices 2014; 6:169-188, doi:10.1002/wsbm. 1260 .

[3] Sotiropoulos F. Computational Fluid Dynamics for Medical Device Design and Evaluation: Are We There Yet? Cardiovasc. Eng. Technol. 2012; 3(2):137-138, doi:10.1007/ s13239-012-0095-5.

[4] Mendez S, Zmijanovic V, Gibaud E, Siguenza J, Nicoud F. Assessing Macroscopic Models for Hemolysis from Fully Resolved Simulations. 4th International Conference on Computational and Mathematical Biomedical Engineering, Nithiarasu P, Budyn E (eds.), CMBE2015 Proceedings, ENS Cachan: France, 2015; 575-578. ISSN 2227-3085.

[5] Hariharan P, Giarra M, Reddy V, Day SW, Manning KB, Deutsch S, Stewart SFC, Myers MR, Berman MR, Burgreen GW, et al.. Multilaboratory Particle Image Velocimetry Analysis of the FDA Benchmark Nozzle Model to Support Validation of Computational Fluid Dynamics Simulations. J. Biomech. Eng. 2011; 133(4):041 002(1-14), doi:10.1115/1.4003440.

[6] Wu X, Moin P, Adrian RJ, Baltzer JR. Osborne Reynolds pipe flow: Direct simulation from laminar through gradual transition to fully developed turbulence. Proc. Natl. Acad. Sci. U.S.A. 2015; 112(26):7920-7924, doi:10.1073/pnas.1509451112. 
[7] Pringle CCT, Duguet Y, Kerswell RR. Highly symmetric travelling waves in pipe flow. Phil. Trans. R. Soc. A 2009; 367(1888):457-472, doi:10.1098/rsta.2008.0236.

[8] Stewart SF, Paterson EG, Burgreen GW, Hariharan P, Giarra M, Reddy V, Day SW, Manning KB, Deutsch S, Myers MR, et al.. Assessment of CFD Performance in Simulations of an Idealized Medical Device: Results of FDA's First Computational Interlaboratory Study. Cardiovasc. Eng. Technol. 2012; 3(2):139-160, doi:10.1007/s13239-012-0087-5.

[9] Passerini T, Quaini A, Villa U, Veneziani A, Canic S. Validation of an open source framework for the simulation of blood flow in rigid and deformable vessels. Int. J. Numer. Meth. Biomed. Engng. 2013; 29(11):1192-1213, doi:10.1002/cnm.2568.

[10] White AT, Chong CK. Rotational invariance in the three-dimensional lattice Boltzmann method is dependent on the choice of lattice. J. Comput. Phys. 2011; 230(16):6367-6378, doi:10.1016/j.jcp.2011.04.031.

[11] Delorme Y, Anupindi K, Frankel SH. Large Eddy Simulation of FDA's Idealized Medical Device. Cardiovasc. Eng. Technol. 2013; 4(4):392-407, doi:10.1007/s1329-013-0161-7.

[12] Janiga G. Large eddy simulation of the FDA benchmark nozzle for a Reynolds number of 6500. Comput. Biol. Med. 2014; 47(Apr.):113-119, doi:10.1016/j.compbiomed.2014.01.004.

[13] Bhushan S, Walters DK, Burgreen GW. Laminar, Turbulent, and Transitional Simulations in Benchmark Cases with Cardiovascular Device Features. Cardiovasc. Eng. Technol. 2013; 4(4):408-426, doi:10.1007/s13239-013-0155-5.

[14] Vreman AW. An eddy-viscosity subgrid-scale model for turbulent shear flow: Algebraic theory and applications. Phys. Fluids 2004; 16(10):3670-3681), doi:10.1063/1.1785131.

[15] Pal A, Anupindi K, Delorme Y, Ghaisas N, Shetty DA, Frankel SH. Large Eddy Simulation of Transitional Flow in an Idealized Stenotic Blood Vessel: Evaluation of Subgrid Scale Models. J. Biomech. Eng. 2014; 136(7):071 009(1-8), doi:10.1115/1.4027610.

[16] Nicoud F, Toda HB, Cabrit O, Bose S, Lee J. Using singular values to build a subgrid-scale model for large eddy simulations. Phys. Fluids 2011; 23(8):085106(1-12), doi:10.1063/1. 3623274 .

[17] Eckhardt B. A Critical Point for Turbulence. Science 2011; 333(192):165-166, doi:10.1126/ science. 1208261.

[18] Avila K, Moxey D, de Lozar A, Avila M, Barkley D, Hof B. The Onset of Turbulence in Pipe Flow. Science 2011; 333(192):447-468, doi:10.1126/science.1203223.

[19] Eckhardt B, Faist H, Schmiegel A. Dynamical systems and the transition to turbulence in linearly stable shear flows. Phil. Trans. R. Soc. A 2008; 366(1868):1297-1315, doi:10.1098/ rsta.2007.2132.

[20] Ng HCH, Monty JP, Hutchins N, Chong MS, Marusic I. Comparison of turbulent channel and pipe flows with varying Reynolds number. Exp. Fluids 2011; 51(5):1261-1281, doi: 10.1007/s00348-011-1143-x.

[21] Mullin T. Experimental Studies of Transition to Turbulence in a Pipe. Annu. Rev. Fluid Mech 2011; 43:1-24, doi:10.1146/annurev-fluid-122109-160652.

[22] Nishi M, Unsal B, Durst F, Biswas G. Laminar-to-turbulent transition of pipe flows through puffs and slugs. J. Fluid Mech. 2008; 614:425-446, doi:10.1017/S0022112008003315.

[23] Eckhardt B, Schneider TM, Hof B, Westerweel J. Turbulence transition in Pipe Flow. Annu. Rev. Fluid Mech 2007; 39:447-468, doi:10.1146/annurev.fluid.39.050905.110308. 
[24] Duguet Y, Willis AP, Kerswell RR. Transition in pipe flow: the saddle structure on the boundary of turbulence. J. Fluid Mech. 2008; 613:255-274, doi:10.1017/S0022112008003248.

[25] Hultmark M, Bailey SCC, Smits AJ. Scaling of near-wall turbulence in pipe flow. J. Fluid Mech. 2010; 649:103-113, doi:10.1017/S0022112009994071.

[26] Mendez S, Gibaud E, Nicoud F. An unstructured solver for simulations of deformable particles in flows at arbitrary Reynolds numbers. J. Comput. Phys. 2014; 256:465-483, doi: 10.1016/j.jcp.2013.08.061.

[27] Mendez S, Chnafa C, Gibaud E, Siguenza J, Moureau V, Nicoud F. YALES2BIO: A Computational Fluid Dynamics Software Dedicated to the Prediction of Blood Flows in Biomedical Devices. 5th International Conference on Biomedical Engineering in Vietnam, IFMBE Proceedings, vol. 46, Toi VV, Phuong THL (eds.), Springer International Publishing: Switzerland, 2015; 7-10, doi:10.1007/978-3-319-11776-82.

[28] Moin P. Advances in Large Eddy Simulation Methodology for Complex Flows. Int. J. Heat Fluid Flow 2002; 23:710-720, doi:10.1016/S0142-727X(02)00167-4.

[29] Moureau V, Domingo P, Vervisch L. Design of a massively parallel CFD code for complex geometries. C. R. Mecanique 2011; 339(2-3):141-148, doi:10.1016/j.crme.2010.12.001.

[30] Chnafa C, Mendez S, Nicoud F. Image-based Large-Eddy Simulation in a realistic left heart. Comput. Fluids 2014; 94:173-187, doi:10.1016/j.compfluid.2014.01.030.

[31] Dubois F, Mozul R. LMGC90. 11e Colloque National en Calcul des Structures, CSMA 2013, Giens, Var: France, 2013; 1-8.

[32] Siguenza J, Mendez S, Ambard D, Dubois F, Jourdan F, Mozul R, Nicoud F. Validation of an immersed thick boundary method for simulating fluid-structure interactions of deformable membranes. J. Comput. Phys. 2016; (in review).

[33] Baya Toda H, Cabrit O, Truffin K, Bruneaux G, Nicoud F. Assessment of subgrid-scale models with a large-eddy simulation-dedicated experimental database: The pulsatile impinging jet in turbulent cross-flow. Phys. Fluids 2014; 26(7):075 108.

[34] Chorin A. Numerical Solution of the NavierStokes Equations. Math. Comput. 1968; 22(8):745-762), doi:10.1090/S0025-5718-1968-0242392-2.

[35] Williamson JH. Low-Storage Runge-Kutta Schemes. J. Comput. Phys. 1980; 35(2):48-56, doi:10.1016/0021-9991(80)90033-9.

[36] Kraushaar M. Application of the Compressible and Low-Mach Number Approaches to Large-Eddy Simulation of Turbulent Flows in Aero-Engines. PhD Thesis, Institut National Polytechnique Toulouse, University of Toulouse, France. Dec 2011. See also URL https://hal .archives-ouvertes.fr/tel-00711480.

[37] Quartapelle L, Selmin V. High-order Taylor-Galerkin methods for non-linear multidimensional problems. Finite Element in Fluids: New Trends and Applications. 1993; 1374-1384.

[38] Malandain M, Maheu N, Moureau V. Optimization of the deflated Conjugate Gradient algorithm for the solving of elliptic equations on massively parallel machines. J. Comput. Phys. 2013; 238:32-47, doi:10.1016/j.jcp.2012.11.046.

[39] Bodony DJ, Lele SK. On using large-eddy simulation for the prediction of noise from cold and heated turbulent jets. Phys. Fluids 2005; 17(8):085 103(1-20), doi:10.1063/1.2001689.

[40] Stewart SF, Hariharan P. Computational Round Robin \#1 (Nozzle). https : //fdacfd.nci. nih.gov/. Accessed: 2015-12-15. 